\title{
Restoring Shank3 in a rostral sensorimotor brainstem nucleus rescues reduced light-evoked behaviors in shank3ab-/- zebrafish.
}

\author{
Robert Kozol \\ Florida Atlantic University \\ David James \\ University of Miami \\ Ivan Varela \\ University of Miami \\ Sureni Sumathipala \\ University of Miami

\section{Stephan Züchner} \\ University of Miami https://orcid.org/0000-0002-8498-5235 \\ Julia Dallman ( $\boldsymbol{\sim}$ j.dallman@miami.edu ) \\ University of Miami https://orcid.org/0000-0002-5839-807X
}

\section{Article}

Keywords: Phelan-McDermid Syndrome, SHANK3 gene, brainstem, hyporeactivity, light-evoked behavior

Posted Date: March 22nd, 2021

DOl: https://doi.org/10.21203/rs.3.rs-83115/v1

License: () (1) This work is licensed under a Creative Commons Attribution 4.0 International License. Read Full License

Version of Record: A version of this preprint was published at Communications Biology on December 17th, 2021. See the published version at https://doi.org/10.1038/s42003-021-02920-6. 


\section{Abstract}

People with Phelan-McDermid Syndrome, caused by mutations in the SHANK3 gene, commonly present with symptoms of sensory hyporeactivity. To investigate how shank3 mutations impact brain circuits and contribute to sensory hyporeactivity, we generated two shank3 zebrafish mutant models. These shank3 mutant models both exhibit hyporeactivity to visual stimuli. Using whole-brain activity mapping, we show that light receptive brain nuclei show normal levels of activity while sensorimotor integration and motor regions are less active in shank3-/- mutants. Specifically rescuing Shank3 in a sensorimotor nucleus of the rostral brainstem is sufficient to rescue shank3-/- mutant hyporeactivity. In summary, reduced sensory responsiveness in shank3-/- mutant is associated with reduced activity across the brain and can be rescued by restoring Shank3 function in the rostral brainstem.

\section{Main Text}

Altered sensory processing is a pervasive but poorly understood symptom in individuals with autism spectrum disorders (ASD) ${ }^{1}$. Sensory symptoms manifest as muted or excessive responses to light, sound, and/or touch. Because of variability in both the presence and presentation of sensory symptoms, gaining a mechanistic understanding of these sensory processing deficits remains a challenge. In contrast to ASD as a whole, genetically defined forms of ASD share similar sensory deficits. For instance, individuals with Phelan McDermid Syndrome (PMS), a syndromic form of ASD, show low sensitivity to pain and reduced responses to auditory and visual stimuli ${ }^{2,3}$. PMS is caused by the loss of function of one copy of the SHANK3 gene, due to either terminal deletions of chromosome $22{ }^{4}$ or SHANK3 point mutations ${ }^{2}$. Here we identify the neurobiological basis of sensory hyporeactivity in shank3 loss-of-function zebrafish models of PMS.

Several animal models of PMS recapitulate muted responses to diverse sensory stimuli: pain in Shank3 mutant mice ${ }^{5}$, sound in Shank 3 mutant rats ${ }^{6}$, and both touch and light in shank3ab mutant zebrafish ${ }^{7,8}$; nonetheless, a brain-wide understanding of these muted responses is lacking. Hyporeactivity in PMS could reflect functional changes that either span the entire brain or are localized to specific brain regions and/or muscle ${ }^{9}$. Zebrafish allow unique experimental approaches to identifying underlying mechanisms because, within the first week of life, larvae have fully functional sensory-motor circuits and produce robust, stereotyped responses to calibrated sensory stimuli ${ }^{10}$ These larval zebrafish have transparent vertebrate brains composed of only 100,000 neurons, allowing unbiased functional approaches to map brain-wide neuronal activity. Moreover, embryonic transplantation can be used to make wildtype-mutant chimeras to test for brain-region-specific functional rescue 11,12 . Here, we use brain-wide activity mapping and transplants to identify and functionally validate brain regions that underlie sensory hyporeactivity to changes in light in zebrafish shank3 mutant models. 
In contrast to the single SHANK3 gene in people, the shank3 gene is duplicated in zebrafish; therefore, to generate zebrafish models of PMS we used CRISPR/Cas9 to mutate both the shank3a and shank3b (shank3ab) gene paralogs. Shank3 proteins are large, $200 \mathrm{kD}$, with multiple isoforms that can be differentially impacted by mutations in different parts of the gene ${ }^{13}$. To capture this complexity, we generated two zebrafish PMS models, shank3abDN with mutations truncating both the Shank3 a and $b$ proteins in the ankyrin repeat domains and shank3abDC with mutations truncating both the Shank3 a and $\mathrm{b}$ proteins near the proline-rich domain ${ }^{13}$ (Fig. 1a; Supplementary Fig. S1, Supplementary Tables 1\&2). These models mimic the most common types of SHANK3 mutations found in people with PMS and, by having two models, we control for genetic background. In mice and humans, Shank3 protein is expressed in glutamatergic granule cells of the cerebellum, colocalizing with the scaffolding protein PSD95. Likewise, in wildtype zebrafish, we show that Shank3 protein colocalizes with PSD-95 in the cerebellum and along ventral neural tracts of the brainstem (Fig. 1b; Supplementary Fig. S2). In contrast, in both shank3abDN-/-and shank3abDC-/-PMS models Shank3 staining is lacking despite intact PSD-95 synaptic puncta (Fig. 1b). These data indicate that the four alleles that underlie the two shank3abDN-/-and shank3abDC-/-models are loss-of-function mutations. Hereafter, we refer to shank3abDN-/-and shank3abDC-/-models are as shank3ab-/-mutant models except in cases that the results differ between the models.

The sensory reactivity of zebrafish shank3ab-/-models was measured by quantifying behavioral changes to a light-based stimulus using the well-established visual motor response $\left(\mathrm{VMR}^{;}{ }^{14}\right)$. The VMR is characterized by dramatic increases in movement in response to sudden transitions from light to darkness (Fig. 1c). Both shank3ab-/-mutant models exhibited reduced VMR responses as quantified by comparing the distance traveled in the thirty seconds before and after the transition from lights-on to lights-off conditions (Fig. 1c \& d; Supplementary Tables 3-8). Muted VMR responses were more pronounced in homozygous shank3ab-/-larvae $(\mathrm{p}<0.001)$ than in heterozygous shank3ab+/larvae $(p<0.05)$. We used the pronounced VMR deficits shank3ab-/-mutants as the basis of all subsequent experiments to determine the mechanistic underpinnings of these altered sensorimotor integration phenotypes.

To identify the neural circuits underlying hyporeactivity in shank3ab mutant models, we used an unbiased brain-wide Mitogen Activated Protein (MAP)-mapping ${ }^{12}$ approach, based on phosphorylation of extracellular signal-regulated kinase (pERK). Because ERK phosphorylation increases when calcium is elevated during action potentials, staining for pERK provides a proxy for neuronal activity (Fig. 2a \& b). Brain regions differentially active between light-on and lights-off conditions were identified by statistically comparing relative ERK signals (pERK/total ERK) in two groups of $15-21$ larvae per group $\left(p<10^{5}\right.$; Fig. $2 \mathrm{c}$ \& d, Supplementary Figs. S3 \& S6). In response to the lights-on stimulus, wild type (WT) and shank3ab-/models showed similarly elevated pERK staining in the optic tectum (green) that receives input from retinal ganglion cells. In response to the lights-off stimulus, WT showed elevated pERK staining in the pineal, the telencephalic pallium and subpallium, the torus semicircularis of the midbrain, brainstem, and spinal cord (magenta). While shank3ab-/-mutant models showed similarly elevated pERK staining in the 
pineal, they showed little or no elevated pERK other brain regions. These VMR brain activity maps in shank3ab-/-models show that sensory brain regions including the pineal, retina, and optic tectum detect changes in light normally, but that downstream brain regions fail to integrate and respond to dark transitions consistent with muted lights-off behavioral responses.

Next we explored whether restoring Shank3 function would be sufficient to rescue hyporeactivity in both shank3abDN-/-and $D C$-/-mutant models. We generated genetically mosaic larvae by transplanting WT cells into otherwise shank3ab mutant embryos at the late gastrula shield stage, $\sim$ six hours postfertilization (Fig. 3a, Supplementary Fig. S5). WT donor cells were deposited in the region of the shank3ab-/-embryo fated to become brainstem. To track the fate of transplanted cells, WT donor Zebrabow embryos expressing dTomato under a ubiquition promoter ${ }^{15}$ were used as the source of WT cells, referred to as ZbT for Zebrabow transplants (Fig. 3). Remarkably, when tested as six-day-old larvae, transplanted ZbT cells were sufficient to rescue shank3ab-/-mutant lights-off reactivity in the VMR assay (Fig. 3 b-d, Supplementary Fig. S5; Supplementary Tables S9-16). To determine ZbT brain regions in common among behaviorally rescued shank3abD:ZbT larvae, we registered shank3abD:ZbT larvae to the Z-brain atlas. We found that the majority of rescued shank3abD:ZbT larvae had integrated ZbT cells in a rostral dorsal glutamatergic brainstem nucleus known in zebrafish brain atlases as vGluT cluster 2 (90.5\%; n=19/21; Supplementary Fig. S6; Supplementary Table S17). Previous studies using whole-brain gCaMP have identified this brainstem nucleus as important in transforming sensory inputs to behavioral responses ${ }^{16,17}$. To control for non-specific transplantation effects, we performed within genotype transplants. WT donor to WT recipient chimeras and shank3abDN-/-donor to shank3abDN-/-recipient chimeras had no effects on VMR behaviors compared to unmanipulated larvae of the corresponding genotype. shank3abDC-/-donor to shank3abDC-/-recipient chimeras had more severe hyporeactivity compared to unmanipulated larvae of the same genotype (Supplementary Figs. S7, Supplementary Tables S18-23). Consistent with the MAP-mapping experiments, these results indicate Shank3ab function in rostral brainstem is sufficient for WT levels of light-evoked activity.

Previous work applying whole brain imaging in zebrafish larvae has highlighted the roles of the cerebellum and rostral brainstem as regions that receive inputs from sensory centers to coordinate the appropriate motor output ${ }^{16-18}$. Brainstem deficits in shank3abDN-/-and $D C$-/-mutants could be due to synaptic deficits and/or altered development. In support of a synaptic role, loss of Shank3 protein in mammalian models is known to decrease glutamate receptor expression, disrupt post-synaptic density composition, and reduce synaptic transmission ${ }^{19}$. Weaker excitatory synaptic responses could therefore explain the failure of sensory brain regions to evoke responses at the levels of both other brain regions and motor behaviors in shank3ab mutant PMS models. Functional deficits could also be due to altered development that could disrupt functional connectivity. Supporting this possibility, global developmental delay has previously been reported in shank3ab zebrafish models ${ }^{7,8}$. Moreover, altered brainstem development has been suggested as the likely basis for multisensory integration and sensory-motor deficits more generally in $A S D^{20,21}$. Such developmental deficits in brainstem regions could help explain the efficacy of rostral sensorimotor brainstem transplants in rescuing VMR behaviors in shank3ab-/- 
mutants in this study. With the recent inclusion of sensory deficits, more clinical research is needed to determine links between changes in the brainstem function and sensory deficits in individuals with autism.

\section{Conclusion}

Brain-wide activity mapping and transplant rescue experiments provide robust evidence that hyporeactivity to light-based stimuli in zebrafish shank3ab mutants is due to functional deficits downstream of sensory reception that can be rescued by restoring wild type Shank3 in the rostral brainstem.

\section{Declarations}

\section{Acknowledgements}

We would like to thank Drs. Sheyum Syed, Kevin Collins, James Baker and Athula Wikramanayake for providing valuable feedback during the writing and editing process. Also, thank you to Dr. Sheym Syed for writing custom MATLAB scripts to analyze raw behavioral data (available on request). To our fish facility manager, Ricardo Cepeda, thank you for keeping the fish happy and healthy.

\section{Funding Statement}

This work was supported by Bridge Funds from the College of Arts and Sciences at the University of Miami and NIH grants NIMH R03MH103857 and NICHD R21HD093021 to JED and an IMSD graduate fellowship from NIH parent grant R25GM076419, HHMI teaching fellowship, and McKnight Dissertation Fellowship to DMJ.

\section{Author Contributions}

R.A.K. and J.E.D conceived the project. R.A.K., D.M.J, S.Z., and J.E.D. wrote the manuscript. R.A.K., D.M.J., I.V. and S.S. conducted experiments and analyzed data. All authors contributed to the editing of the final intellectual product.

\section{Ethics Declarations}

The authors declare no conflicts of interest.

\section{References}

1. Robertson, C.E. \& Baron-Cohen, S. Sensory perception in autism. Nat Rev Neurosci $18,671-684$ (2017).

2. De Rubeis S, S.P., Durkin A, Weissman J, Muratet F, Halpern D, Trelles MdP, Frank Y, Lozano R, Wang AT, Holder Jr JL, Betancur C, Buxbaum JD, Kolevzon A. Delineation of the genetic and clinical 
spectrum of Phelan-McDermid syndrome caused by SHANK3 point mutations. Molecular Autism (2018).

3. Mieses, A.M., et al. Brief Report: Sensory Reactivity in Children with Phelan-McDermid Syndrome. $J$ Autism Dev Disord 46, 2508-2513 (2016).

4. Phelan, K. \& McDermid, H.E. The 22q13.3 Deletion Syndrome (Phelan-McDermid Syndrome). Mol Syndromo/ 2, 186-201 (2012).

5. Han, Q., et al. SHANK3 Deficiency Impairs Heat Hyperalgesia and TRPV1 Signaling in Primary Sensory Neurons. Neuron 92, 1279-1293 (2016).

6. Engineer, C.T., et al. Shank3-deficient rats exhibit degraded cortical responses to sound. Autism Res 11, 59-68 (2018).

7. Kozol, R.A., et al. Two knockdown models of the autism genes SYNGAP1 and SHANK3 in zebrafish produce similar behavioral phenotypes associated with embryonic disruptions of brain morphogenesis. Hum Mol Genet 24, 4006-4023 (2015).

8. Liu, C.X., et al. CRISPR/Cas9-induced shank3b mutant zebrafish display autism-like behaviors. Mol Autism 9, 23 (2018).

9. Lutz, A.K., et al. Autism-associated SHANK3 mutations impair maturation of neuromuscular junctions and striated muscles. Sci Transl Med 12 (2020).

10. Portugues, R., Severi, K.E., Wyart, C. \& Ahrens, M.B. Optogenetics in a transparent animal: circuit function in the larval zebrafish. Curr Opin Neurobio/ 23, 119-126 (2013).

11. Wee, C.L., et al. A bidirectional network for appetite control in larval zebrafish. Elife 8 (2019).

12. Randlett, O., et al. Whole-brain activity mapping onto a zebrafish brain atlas. Nat Methods 12 , 10391046 (2015).

13. James, D.M., et al. Intestinal dysmotility in a zebrafish (Danio rerio) shank3a;shank3b mutant model of autism. Mol Autism 10, 3 (2019).

14. Burgess, H.A. \& Granato, M. Modulation of locomotor activity in larval zebrafish during light adaptation. J Exp Bio/ 210, 2526-2539 (2007).

15. Woo, K. \& Fraser, S.E. Order and coherence in the fate map of the zebrafish nervous system. Development 121, 2595-2609 (1995).

16. Privat, M., et al. Sensorimotor Transformations in the Zebrafish Auditory System. Curr Bio/ 29, 40104023 e4014 (2019).

17. Ahrens, M.B., et al. Brain-wide neuronal dynamics during motor adaptation in zebrafish. Nature $\mathbf{4 8 5}$, 471-477 (2012).

18. Chen, X., et al. Brain-wide Organization of Neuronal Activity and Convergent Sensorimotor Transformations in Larval Zebrafish. Neuron 100, 876-890 e875 (2018).

19. Peca, J., et al. Shank3 mutant mice display autistic-like behaviours and striatal dysfunction. Nature 472, 437-442 (2011). 
20. Dadalko, O.I. \& Travers, B.G. Evidence for Brainstem Contributions to Autism Spectrum Disorders. Front Integr Neurosci 12, 47 (2018).

21. Fatemi, S.H., et al. Consensus paper: pathological role of the cerebellum in autism. Cerebellum 11, 777-807 (2012).

\section{Figures}

a

\section{Human SHANK3}
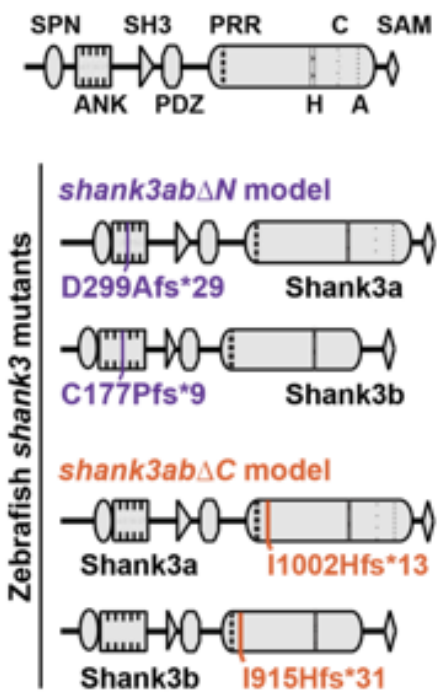

C

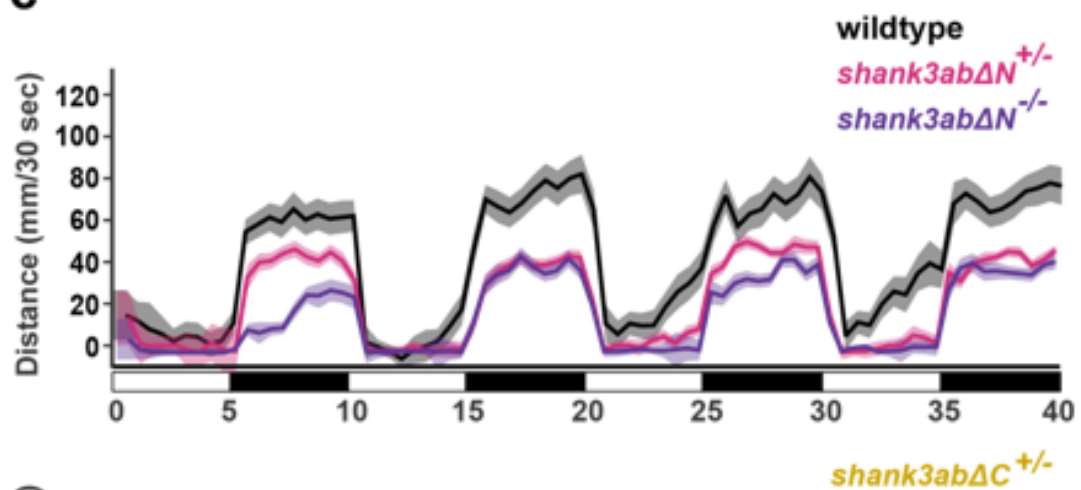

ฮु

b

shank3ab $\Delta C^{-1-}$

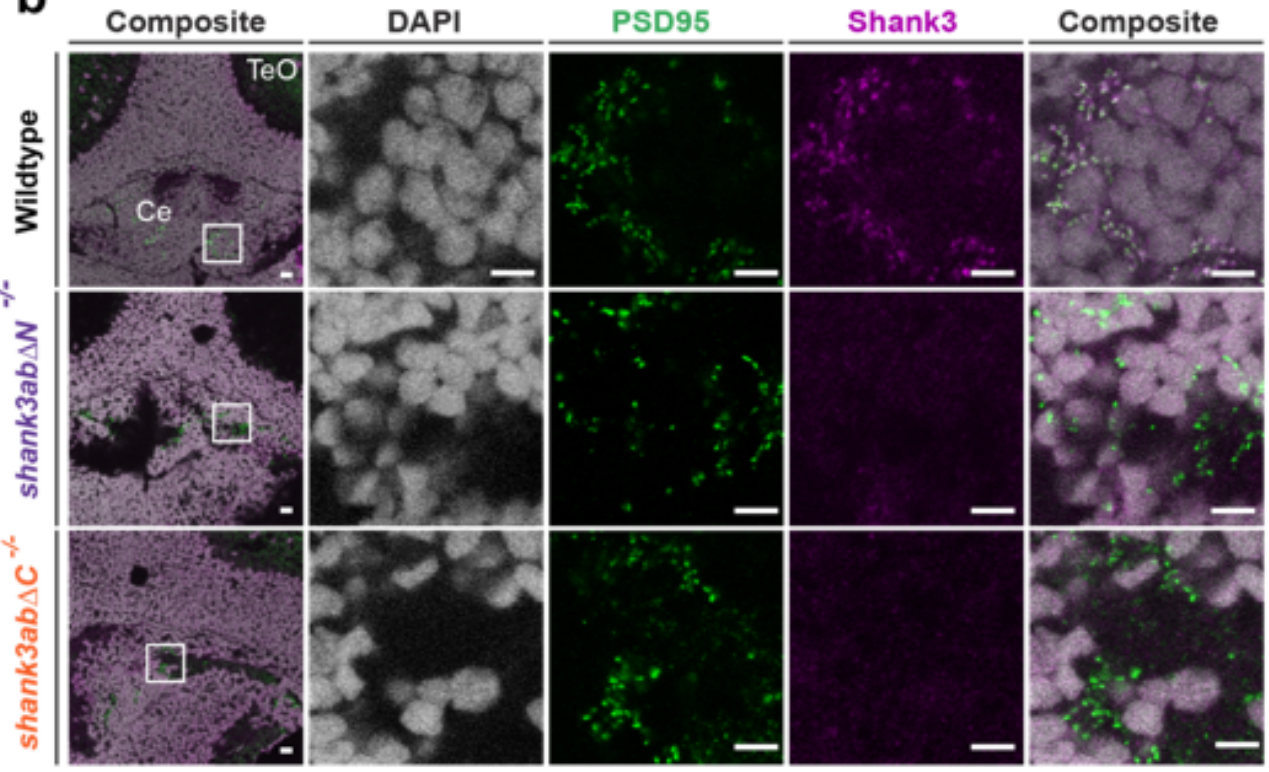

d

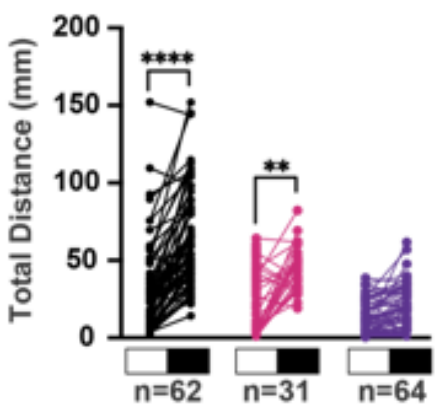

e 30 secOff
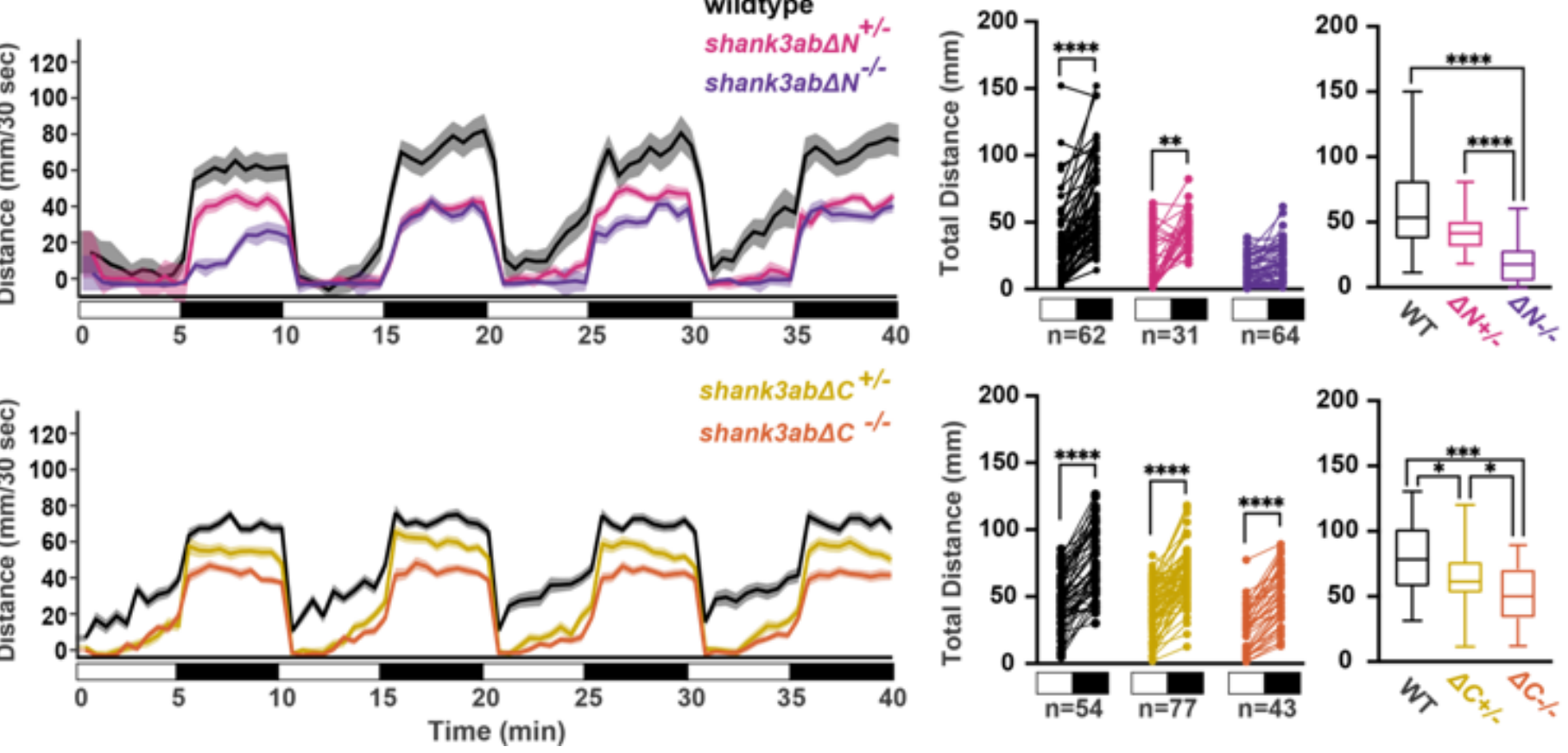
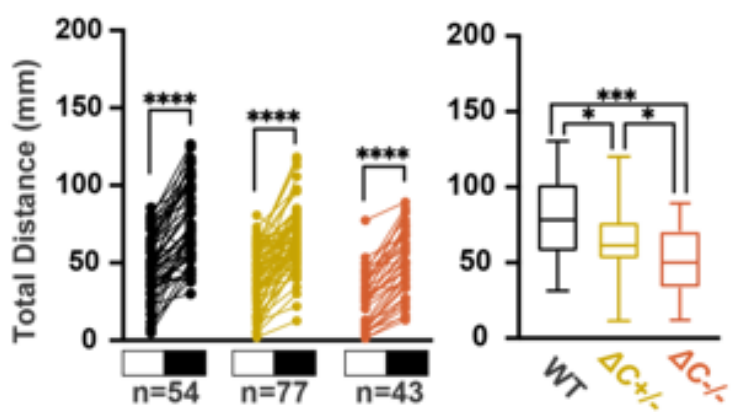

Figure 1 
Two independent shank3ab mutant models are hyporeactive to lights-off stimuli. (a) Shank3 protein diagrams of human SHANK3 and duplicated zebrafish Shank3a and Shank3b show where in the zebrafish proteins four independent CRISPR-Cas 9 indel alleles introduce frameshift mutations. Protein interaction domains indicated in human SHANK3 are more highly conserved in zebrafish Shank3a than Shank3b (SPN=Shank/ProSAP N-terminal, ANK=ankyrin repeats, SH=SRC Homology 3, PDZ=postsynaptic density protein/disc large/zonula occludens-1, PRR=proline-rich region that includes interaction domains with $\mathrm{H}=$ Homer, $\mathrm{C}=$ cortactin, $\mathrm{A}=$ actin binding protein 1 , and $\mathrm{SAM}=$ sterile alpha motif). Each shank3ab $\Delta \mathrm{N}$ (purple) and shank3ab $\Delta \mathrm{C}$ (orange) mutant model has similar mutations in Shank3a and Shank3b paralogs: shank3ab $\Delta \mathrm{N}$ mutations are in ankyrin repeat regions and shank $3 a b \Delta \mathrm{C}$ mutations are in the proline-rich region. (b) Coronal cryosections from $6 \mathrm{dpf}$ larvae were stained with antibodies against the glutamatergic post-synaptic scaffolding proteins PSD-95 and Shank3. Synapses in wildtype cerebellar (Ce) stain for both PSD-95 and Shank3 puncta that are colocalized, compared to shank3ab mutants that stain for PSD-95, but not Shank3. TeO= Optic Tectum; Scale bars represent $10 \mu \mathrm{m}$. (c) Visual motor responses (VMR) are shown as a line-graphs of median distance traveled in 30 seconds +/SE to four cycles of lights-on to lights-off transitions. White and black boxes below the $x$-axis indicate alternating lights-on and lights-off, respectively. Sample sizes are indicated below the Paired dot plots and apply to plots in c, d, and e. (d) Paired dot plots compare median swimming distances per larva of the four light transitions in the 30 seconds before and after the lights-on to lights-off transition. Within genotype comparisons were conducted using Dunn-Bonferroni p-value corrected t-tests (e) Box plots compare distance traveled during the first 30 seconds of dark between WT and shank3ab mutant models. Boxes denote the median, $1 \mathrm{st}$ and 3rd quartile, while whiskers represent the minimum and maximum values. Groups were statistically compared using a Kruskal-Wallis ANOVA, and when statistically significant, were followed by a Dunn's multiple comparison test. P value asterisks represent; $p<0.05$ - *, $p<0.01-* \star, p<0.001-\star \star \star, p<0.0001-\star \star \star \star$. 
a
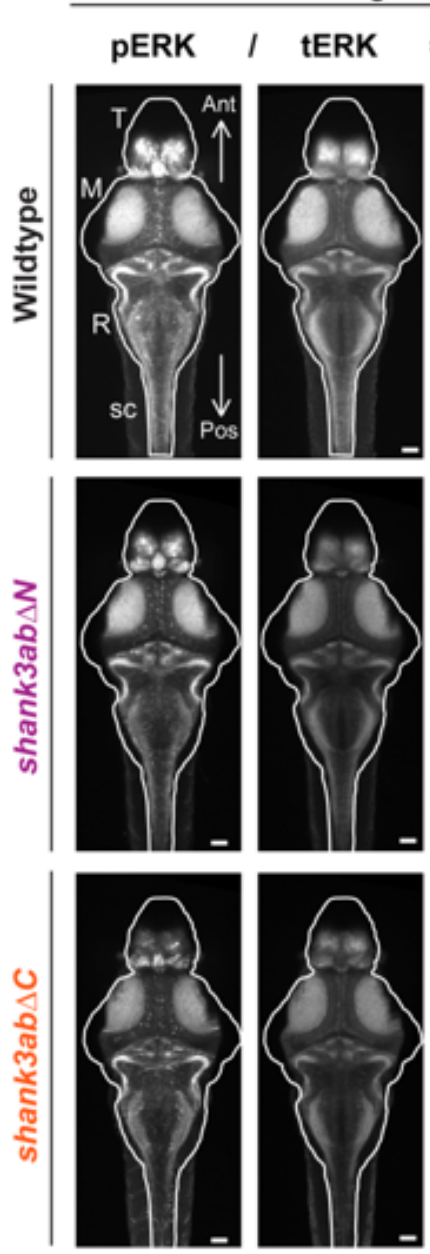

b
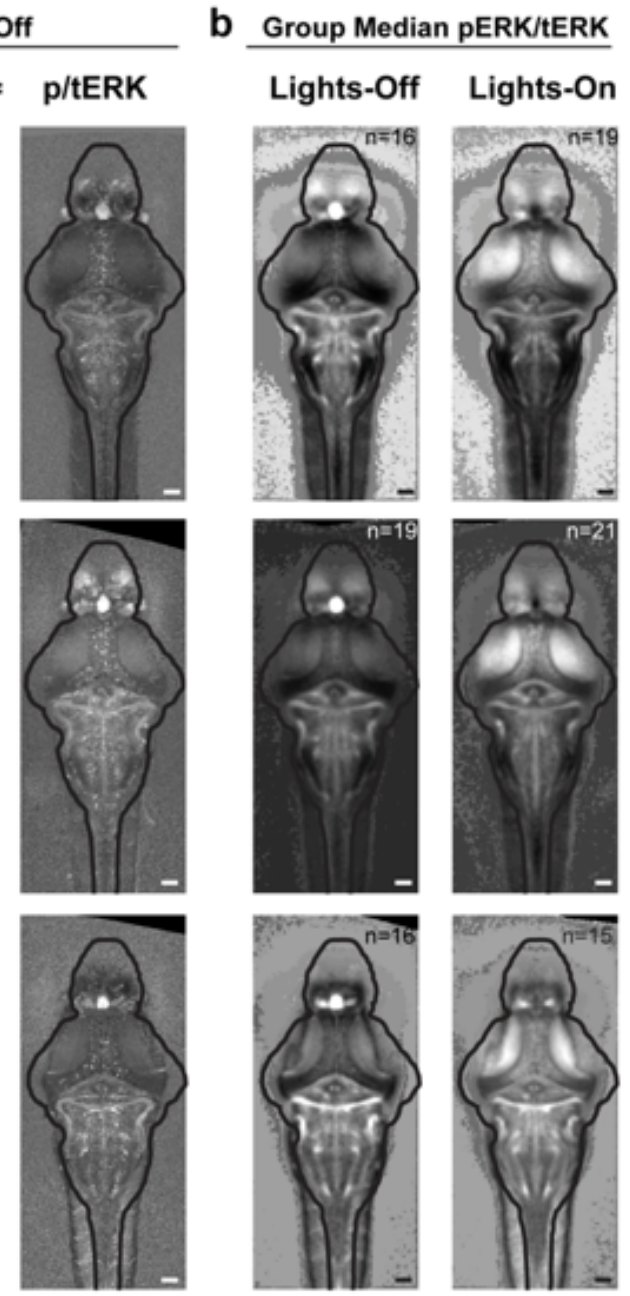
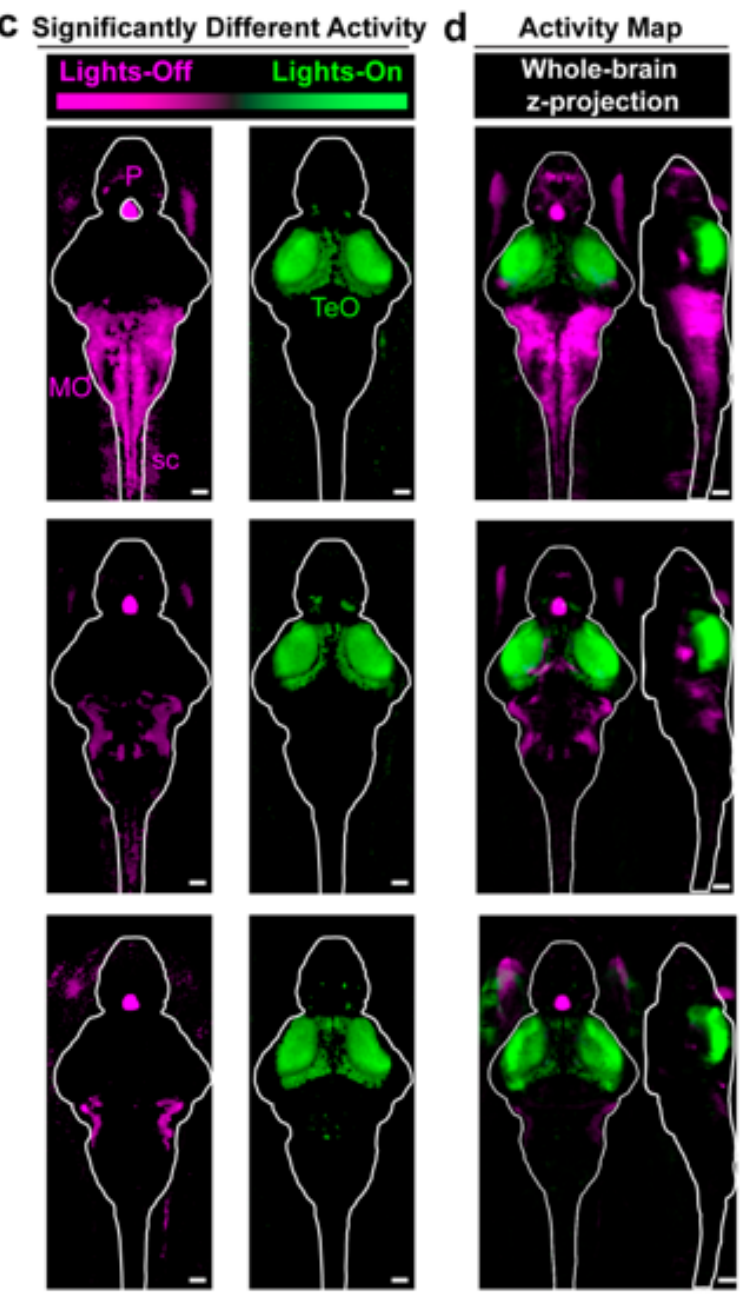

Figure 2

Brain-wide neural activity mapping reveals shank3ab $\Delta-/-$ mutant models sense light normally but fail to activate downstream brain regions underlying sensorimotor integration. Brain-wide activity maps were generated by using phosphorylated-ERK (pERK) antibody staining as a proxy for neuronal activity. (a) Individual larval stacks were registered for use with the Z-brain atlas and MAP-mapping matlab scripts (Randlett et al. 2015, Engert lab) Individual pERK stacks left were then divided by total-ERK (tERK; middle), providing normalized pERK/tERK signal (right). (b) Median p/tERK values were then calculated for every voxel within the brain for each genotype and light condition ( $n=15-21)$. (c) Mann-Whitney U z-scores were calculated, comparing lights-off and lights-on, with magenta indicating significantly increased activity during the transition to lights-off (e.g. Medulla Oblongata, MO) and green indicating significantly increased activity during the transition to lights-on (e.g. Optic Tectum, TeO). Regions within the brain that are black are not statistically significant at the level of $P<105$. (d) In comparison to wildtype, shank3ab $\Delta-/$ - mutant models respond to the lights-off condition (magenta) with activation of their pineal $(P)$, but fail to show significant activation in the MO and spinal cord (sc). (a-c) All images are 20 uM dorsal z-projections. (d) Whole brain z- and x-projections. Sample sizes (n=lights-on/lights-off); wildtype $(n=16 / 19)$, shank3ab $\Delta N(n=19 / 21)$ and shank3ab $\Delta C(n=16 / 15)$. Scale bars $=50 \mu m$ 
a

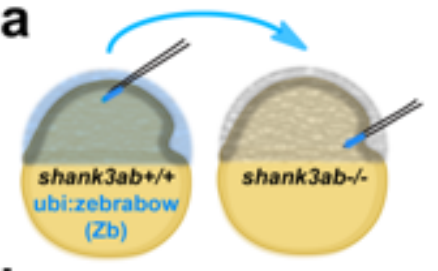

b

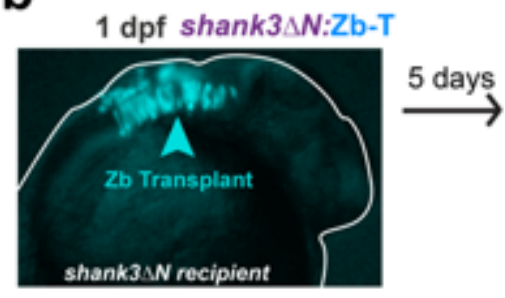

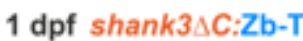

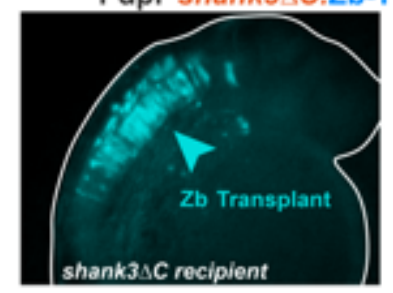

d

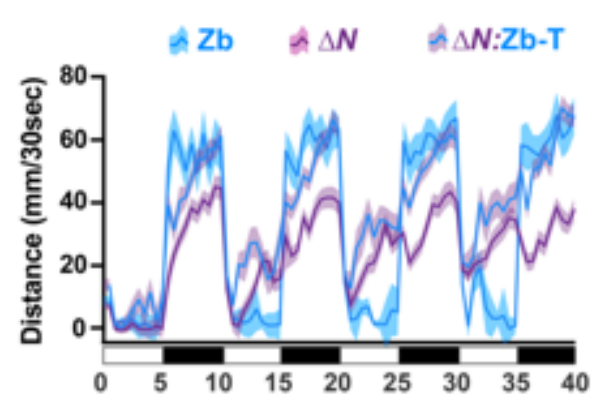

$\Delta \mathrm{Zb} \Leftrightarrow \Delta C$ 스:Zb-T

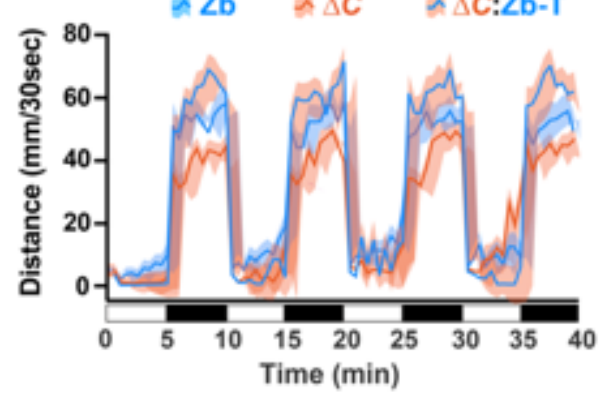

C shank3 $\Delta N: Z b-T$
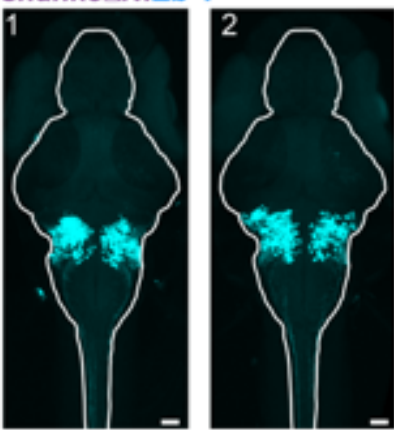

shank3AC:Zb-T
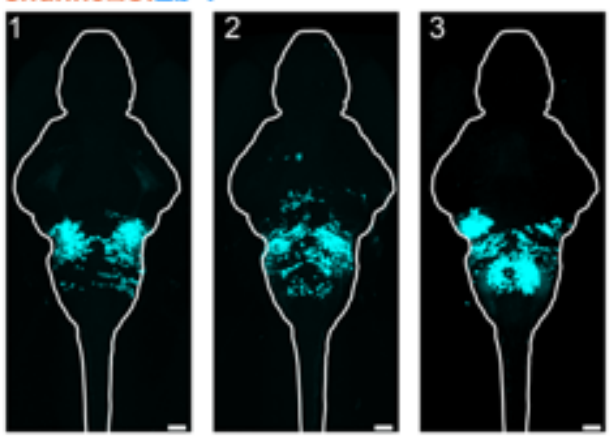

e

30 sec pre- and post- Off

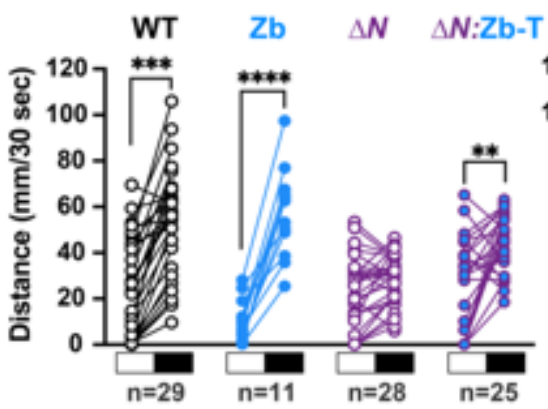

WT Zb $\triangle C \quad \triangle C: Z b-T$

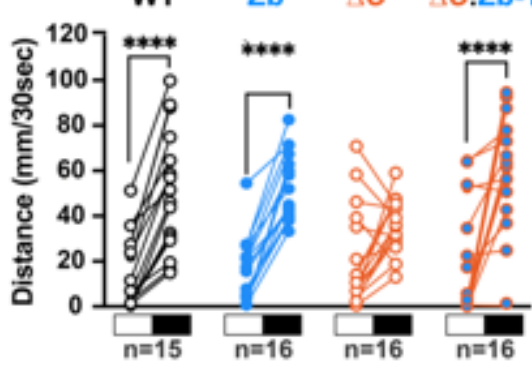

Zb-T Pixel Intensity
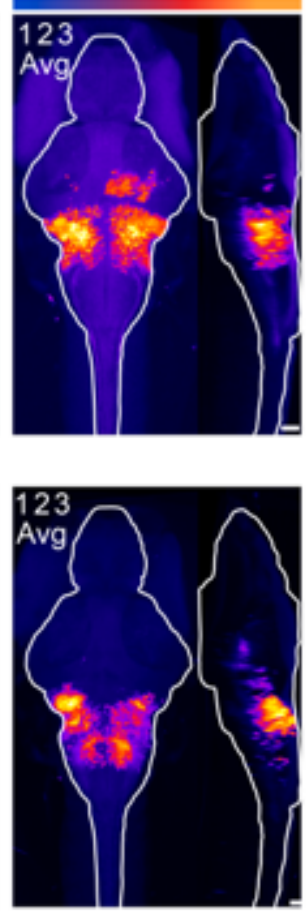

f

First 30 sec Off
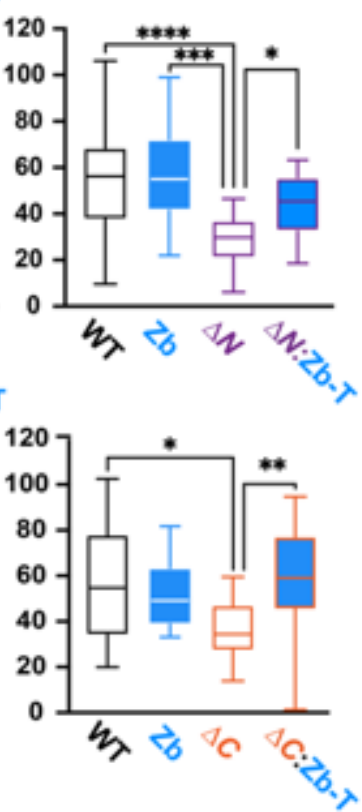

\section{Figure 3}

Hyporeactivity is rescued in both $\Delta \mathrm{N}$ and $\Delta \mathrm{C}$ shank3 mutant models by restoring wild-type shank3ab positive neurons in dorsal/rostral glutamatergic brainstem nuclei. (a) A cartoon above shows how cells from wild type donor embryos marked by a ubiquitously expressed dTomato fluorescent protein (ubi:zebrabow) are transplanted into the presumptive hindbrain of shank3ab-/- mutant recipient embryos at mid-gastrulation stages. (b) Chimeric embryos at 1 day post-fertilization (dpf), with donor cells 
expressing the fluorescent protein (false-colored in cyan) in recipient shank3ab $\Delta \mathrm{N}-/$ - or shank3ab $\Delta \mathrm{C}-/-$ embryos. Chimeric six-day-old larvae (shank3ab-/-:Zb-T) were imaged to determine the fate of the transplanted cells. (c) Confocal images of chimeric larvae at $6 \mathrm{dpf}$ following behavioral screening, demonstrating transplanted cells in rescued larvae populate the dorsal/rostral brainstem nuclei. Individual larvae are numbered 1-3, with the three averaged in the right most stack. Scale bars $=50 \mu \mathrm{m}$. (d) VMR line graphs with ample sizes are indicated below the paired dot plots and apply to plots in d, e, and $\mathrm{f}$. and (e) paired-plots show lights-off behavioral phenotypes are rescued in both shank3ab $\Delta-/-$ mutant models with wild-type-derived brainstems (shank3ab $\Delta-/-: \mathrm{Zb}-\mathrm{T}$ ). Within shank3 model comparisons were conducted using Dunn-Bonferroni p-value corrected t-tests. (f) Box plots displaying median swimming distances for individuals following the first 30-seconds following lights-off. Individual values are medians representing all four lights-off transitions for individual larvae. Boxes denote the median, 1 st and 3 rd quartile, while whiskers represent the minimum and maximum values. Groups were statistically compared using Kruskal-Wallis one-way ANOVA, and when statistically significant, followed

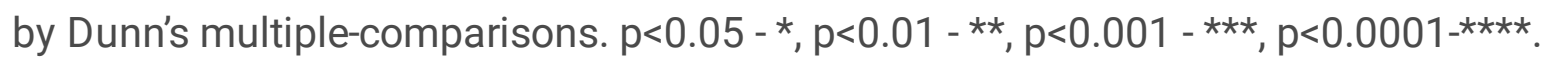

\section{Supplementary Files}

This is a list of supplementary files associated with this preprint. Click to download.

- SupplementaryMaterial.docx 